\title{
Numerical Analysis of the Unsteady Subsonic Flow around a Plunging Airfoil
}

\author{
Kyungwhan Lee* \\ Sunchon National University, Sunchon, Korea \\ Jaesoo Kim** \\ Chosun University, Gwangju, Korea
}

\begin{abstract}
Much numerical and experimental research has been done for the flow around an oscillating airfoil. The main research topics are vortex shedding, dynamic stall phenomenon, MAV's lift and thrust generation. Until now, researches mainly have been concentrated on analyzing the wake flow for the variation of frequency and amplitude at a low angle of attack. In this study, wake structures and acoustic wave propagation characteristics were studied for a plunging airfoil at high angle of attack. The governing equations are the Navier-Stokes equation with LES turbulence model. OHOC (Optimized High-Order Compact) scheme and 4th order Runge-Kutta method were used. The Mach number is 0.3 , the Reynolds number is , and the angle of attack is from $20^{\circ}$ to $50^{\circ}$. The plunging frequency and the amplitude are from 0.05 to 0.15 , and from 0.1 to 0.2 , respectively. Due to the high resolution numerical method, wake vortex shedding and pressure wave propagation process, as well as the propagation characteristics of acoustic waves can be simulated. The results of frequency analysis show that the flow has the mixed characteristics of the forced plunging frequency and the vortex shedding frequency at high angle of attack.
\end{abstract}

Key words: Plunging Airfoil, OHOC(Optimized High Order Compact ) Scheme, Frequency Analysis, Subsonic Unsteady Flow

\section{Introduction}

Much research has been conducted for the unsteady flow around an oscillating airfoil. The main flow characteristics are vortex shedding of the wake, dynamic stall phenomenon, thrust generation of the insect and the whales, lift and thrust generation of MAV. The early stages, theoretical and experimental studies have shown that a certain combination of oscillation frequency and amplitude generates the thrust on the airfoil. Major motion characteristics of the oscillating airfoil are divided into pitch oscillation due to the changes of pitch angle, plunging oscillation due to vertical movement, and flapping oscillation which is the motion combining pitch angle and plunging motion. Each of these oscillations has been analyzed separately due to the complex characteristics of flow. Major factors that affect the flow characteristics are the Reynolds number, frequency, and trailing edge oscillation amplitude [1,2].

The Knoller \& Benz effect was discovered in the early 1990s, in which the flapping airfoil generates aerodynamic thrust force $[1,2]$. Birnbaum[3] introduced the reduced frequency $\mathrm{k}$ that can characterize the flight efficiency using the linear potential theory, and identified that the vortex shedding in the trailing edge is an important factor. Garrick [4] conducted research to obtain the thrust and propulsive efficiency through a certain combination of oscillation frequency and amplitude, using the potential flow analysis. Jone, et al. [5] and Lai \& Platzer [6] numerically studied that the wake may appear either as Karman vortex street or as reversed Karman vortex street depending on the frequency and the amplitude of plunging airfoil, and drag or thrust is generated respectively. Taylor et al. [7] discovered the range of Strouhal
This is an Open Access article distributed under the terms of the Creative Commons Attribution Non-Commercial License (http://creativecommons.org/licenses/by$\mathrm{nc} / 3.0 /$ which permits unrestricted non-commercial use, distribution, and reproduction in any medium, provided the original work is properly cited.

\footnotetext{
(c) * Professor

** Professor, Corresponding author: jsckim@chosun.ac.kr
} 
number by analyzing the flapping cycle and the amplitude through various experiments on insects. Young \& Lai [8] showed that, depending on the reduced frequency at low Reynolds number, the aerodynamic characteristics appear differently according to the leading and the trailing edge separation. Rival \& Tropea [9] demonstrated that the reduced frequency is a major variable in the pure pitching and the pure plunging flow through a dynamic stall process at low Reynolds numbers. Moryossef \& Levy [10] performed a study of the ground effect of the oscillating airfoil, and showed that it has more impact at a low reduced frequency and the phase angle of lift is quite different from the case in free stream. Lai et al. [1] summed up all the studies conducted so far, which range from pure plunging to complex flapping flow.

Most studies focused on the analysis of basic wake characteristics by frequency and amplitude at a low angle of attack. However, this study aims to the pressure and acoustic wave propagation characteristics around the pure plunging airfoil at high angle of attack. The Navier-Stokes equation was used with the LES turbulence model for the governing equation $[11,12]$. A high-resolution numerical method of Optimized High-Order Compact (OHOC) scheme was used for the numerical method [13] and the 4th-order Runge-Kutta technique was used for the time derivatives. Using the OHOC, it was possible to simulate the acoustic wave propagation characteristics, as well as the wake vortex shedding and the pressure wave propagation process.

The flow around oscillating NACA0012 airfoil was analyzed for the angle of attack of $10^{\circ} \sim 50^{\circ}$ at the Mach number of 0.3 and $R e=10^{5}$. The plunging frequency is from 0.05 to 0.15 and the amplitude is from 0.1 to 0.2 . According to the frequency analysis of lift coefficients, the flow characteristics are determined by the principal oscillation frequencies due to the forced plunging oscillation and the large separated flow on the airfoil surface.

\section{The Governing Equations and the Numeri- cal Methods}

The two-dimensional unsteady compressible NavierStokes equation non-dimensionalized in the generalized coordinates was used, as in equation (1).

$$
\frac{\partial \hat{Q}}{\partial t}+\frac{\partial \hat{E}}{\partial \xi}+\frac{\partial \hat{F}}{\partial \xi}=\frac{\partial \hat{E}_{V}}{\partial \xi}+\frac{\partial \hat{F}_{V}}{\partial \eta}+S
$$

where

$$
S=Q\left[\frac{\partial}{\partial t}\left(\frac{1}{J}\right)+\frac{\partial}{\partial \xi}\left(\frac{\xi_{t}}{J}\right)+\frac{\partial}{\partial \eta}\left(\frac{\eta_{t}}{J}\right)\right]+E\left[\frac{\partial}{\partial \xi}\left(\frac{\xi_{x}}{J}\right)+\frac{\partial}{\partial \eta}\left(\frac{\eta_{x}}{J}\right)\right]+F\left[\frac{\partial}{\partial \xi}\left(\frac{\xi_{y}}{J}\right)+\frac{\partial}{\partial \eta}\left(\frac{\eta_{y}}{J}\right)\right]
$$

Non-dimensional parameters are chord length (L), freestream velocity(U) and freestream density $(\rho)$. The characters of $t, \xi$ and $\eta$ represent time and generalized coordinates. $\hat{Q}$ is a non- dimensional flux vector that has transformed in the generalized coordinates, $\hat{E}, \hat{F}, \hat{E}_{V}$, and $\hat{F}_{V}$ represent the nonviscous flux vectors and the viscous flux vectors in each direction. The mesh movements were considered in the conversion matrices for the oscillating airfoil. S denotes the terms of geometric conservation law for a high order scheme.

In order to maintain high order and high resolution for the time derivatives, Runge-Kutta's 4th order scheme was used. For the space derivatives, 4th-order accuracy OHOC (Optimized High-Order Compact) scheme was used [13]. The tri- or penta-diagonal matrix based on 7 points was used to obtain the derivatives of each physical property, as in equation (2). Due to the characteristics of central difference scheme, there occurred truncation and phase errors. In order to minimize such errors, $\alpha, \beta$ and $\alpha_{n}$ coefficients proposed by Kim and Lee [13] was used.

$$
\beta f^{\prime}{ }_{i-2}+\alpha f^{\prime}{ }_{i-1}+{f^{\prime}}_{i}+\alpha f^{\prime}{ }_{i+1}+\beta f^{\prime}{ }_{i+2}=\sum_{n=1}^{3} a_{n}\left(f_{i+n}-f_{i-n}\right)
$$

It is possible to achieve a high resolution for space with the OHOC scheme, but it is impossible to exactly reproduce the characteristics of the wave propagation, as with the upwind scheme. The dissipation and diffusion errors are generated due to the characteristics of central difference scheme. They significantly affect the numerical stability. The adaptive nonlinear artificial dissipation model proposed by Kim and Lee [13] was used to enhance numerical stability.

We used O-type meshes concentrated around the airfoil where the changes of flow properties are expected to be large. The number of 280x91meshes shown in Fig.1 was used, based on the computational tests of several mesh

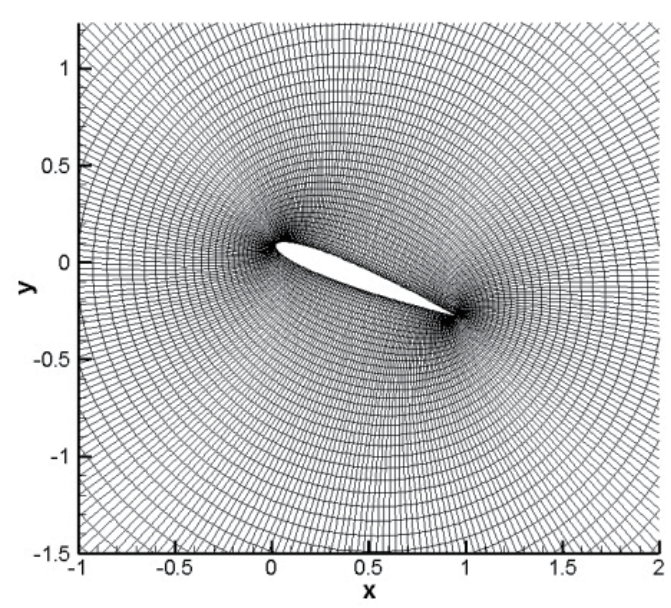

Fig. 1. Computational meshes around airfoil 
systems. The meshes were moved with the moving airfoil surface within the zone of 20 times the chord length of airfoil by algebraic computation. The fixed meshes were used in other zone of 40 times the chord length.

When acoustic waves reach the boundary surface at subsonic flow, physical and non-physical reflected waves are generated. While passing through the boundary, the physical reflected waves have long wavelength but they do not affect numerical stability. However, the non-physical reflected waves have short wavelength and they generate unnecessary numerical instability due to the numerical derivatives at the boundary. These cause numerical instability in the entire computational domain. Particularly, when the turbulent flow or vortices pass through the boundary, highly nonphysical reflected waves occur. Generally, in order to inhibit

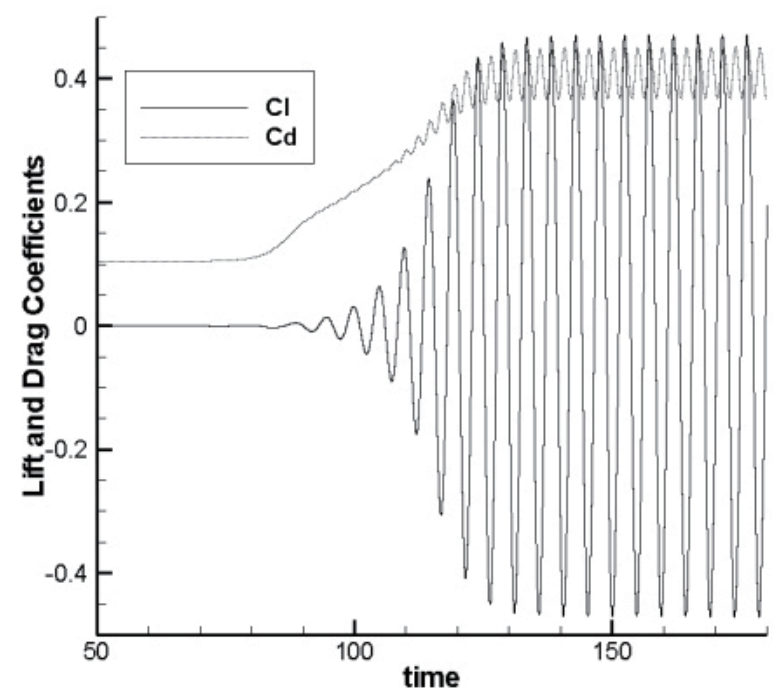

Fig. 2. (a) Lift and drag coefficients

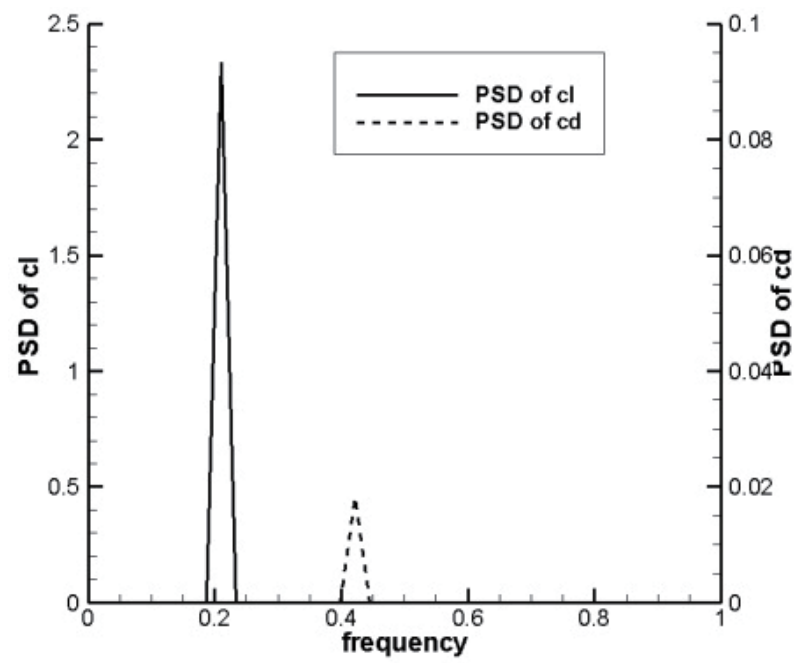

Fig. 2. (b) PSD distribution of lift and drag coefficients non-physical reflection that occurs at the boundary, inflow and outflow conditions according to the eigenvalues are used. However, the characteristic boundary conditions are not sufficient to inhibit non-physical reflection. In this study, the zonal characteristic boundary conditions proposed by Sandberg et al. [14] were applied to minimize the effect of non-physical reflected waves on the computational domain. The zonal characteristic boundary condition was developed on the basis of general local characteristic boundary conditions.

\section{Results and Discussion}

The results have been compared to that of other studies for the flow around a stationary circular cylinder and NACA0012 airfoil to verify the calculations. The results calculated by the same numerical method for the flow around a stationary circular cylinder were compared to other results in Kim and Kim[15]. Its contents briefly are summarized as follows. The flow conditions are Mach number of 0.3 and $\mathrm{Re}=10^{3}$. The Strouhal number $\left(S_{S}\right)$ of 0.21 is clearly shown by the FFT analysis of the lift coefficient, without other frequency disturbance. The value is very similar to $S_{S}$ of 0.21 by Blake [16] and $S_{S}$ of 0.206 by Williamson [17]. The frequency number of drag coefficients is double that of the Strouhal number of lift coefficients.

The flow around NACA0012 airfoil was analyzed for the angle of attack of $10^{\circ} \sim 50^{\circ}$ in the flow condition of Mach number of 0.3 and $\mathrm{Re}=10^{5}$. For this flow, the results calculated by the present numerical method were compared to other results in Yoo and Kim[18]. The comparison can be summarized as follows. The flow of the steady state free stream develops into a periodic unsteady flow due to the

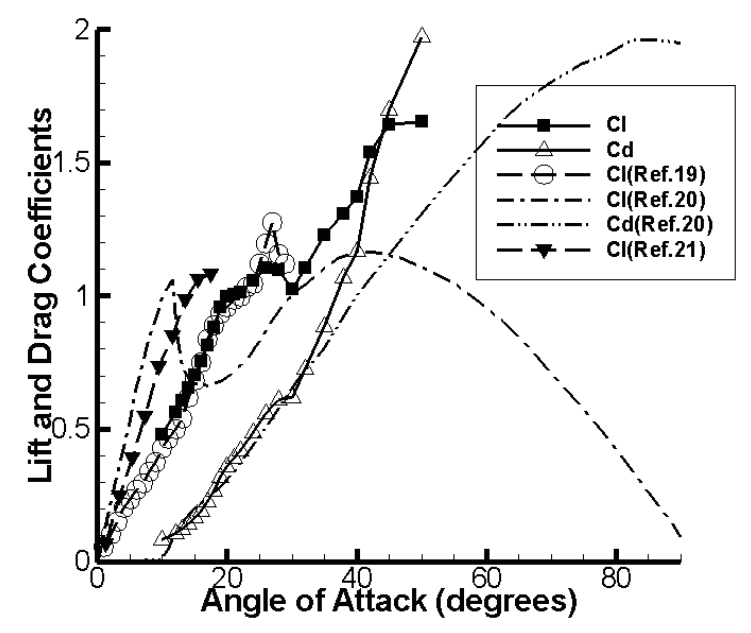

Fig. 3. Lift and drag coefficients for NACA 0012 
generation of the vortex on the surface of the airfoil through the initial transient process, depending on the angle of attack. The mean lift and drag coefficients are compared with other calculations and experimental data in Fig. 3 to verify the calculations. The coefficients are very similar to those of other data at an angle of attack less than $25^{\circ}$ before dynamic stall occurs. Though the lift coefficients differ from each other, the angle of attack where dynamic stall occurs is very similar. The calculated results were compared to the numerical results by Liu, et al. [19] and the experimental results by Critzos, et al. [20] who measured the lift and the drag coefficients at the angles of attack from $0^{\circ}$ to $180^{\circ}$. The slope of lift coefficient variation of present calculation is lower than the experimental data at the region of low angle of attack. The reason may be the effect of low Reynolds number. The result by Craig, et al. [21] who conducted their experiment at a low Reynolds number $\left(3.1 \times 10^{5}\right)$ also shows a low slope of lift coefficient variation. The Reynolds number applied in this study is $10^{5}$ which is higher than $\mathrm{Re}=10^{3}$ of Liu, et al. [19]. The fact, that the slope of lift coefficient variation becomes low due to the effect of the Reynolds number, can also be confirmed with the results by Hoerner \& Borst [22]. The drag coefficients look close to the experimental data before the dynamic stall. After the stall zone, its value becomes larger together with the lift coefficients. In the results of Critzos, et al. [20] and the present results, as the angle of attack passes through the initial zone of dynamic stall, the mean lift and drag drastically increases. The liftdrag ratio becomes one at one angle of attack between $45^{\circ}$ and $50^{\circ}$, and the drag coefficient rapidly increases while the lift coefficient decreases. Although the values of the lift and the drag coefficient may significantly vary due to the inaccuracy of the numerical prediction in the dynamic stall zone and the difference of flow conditions, the results seem

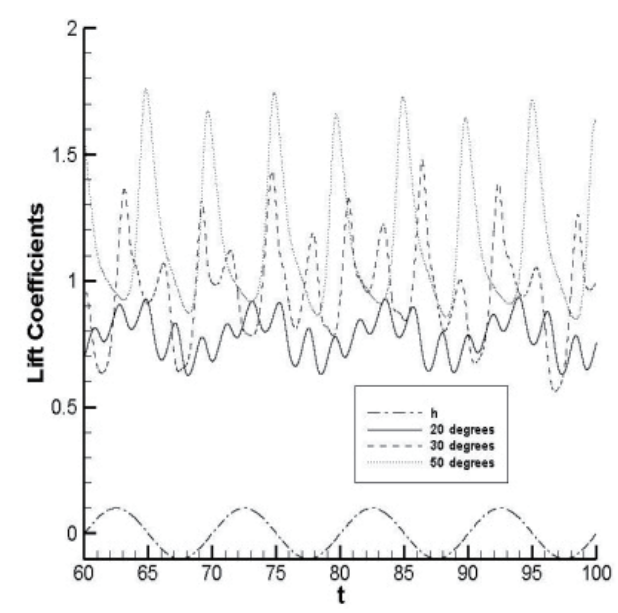

Fig. 4. Lift coefficients for the cases of $h_{0}=0.1$ and $f_{0}=0.1$ to have simulated the flow characteristics well.

The calculation cases to show the flow characteristics by the plunging oscillation are Mach number of 0.3, Reynolds number of $1.0 \times 10^{5}$, and the mean angle of attack of $20^{\circ}$ to $50^{\circ}$. The airfoil position was applied as $h=h_{0} \sin (k t)$, where $k\left(=2 \pi h f_{0}\right)$ and $f_{0}$ are the plunging amplitude, the reduced frequency and the non-dimensional frequency, respectively. The mean angle of attack means the mean angle between the free stream velocity and the chord line. The angle of attack is changed due to the plunging motion each time. The plunging frequency is from 0.05 to 0.15 and the amplitude is from 0.1 to 0.2 .

Variations of the lift and drag coefficients over time are shown in Fig. 4 , when the amplitude $h_{0}=0.1$ and the non-dimensional frequency $f_{0}=0.1$. The results converge to the periodic steady state with the mixed frequency characteristics by the forced oscillation and the vortex shedding in the wake. Fig. 5 and Fig. 6 show the pressure and the entropy distribution when $h_{0}=0.1, f_{0}=0.1$ and the angle of attack of $\alpha=30^{\circ}$. The figures show the acoustic waves that propagate with long wavelength to the entire area and the pressure waves that flow with short wavelength along with the vortex. That is, the acoustic wave propagates to the entire area with a long wavelength and the pressure wave flows along with the vortex within one acoustic wave behind the airfoil. In the pressure and the entropy $s\left(=p / \rho^{r}\right)$ distributions, the wave propagation by the plunging motion is shown, as well as the waves flowing with the vortices in the wake flow. There are three type characteristics of waves. The first one is long-range acoustic waves generated by the airfoil as shown in the pressure distribution. The second one is shortrange waves directly generated by the plunging movement

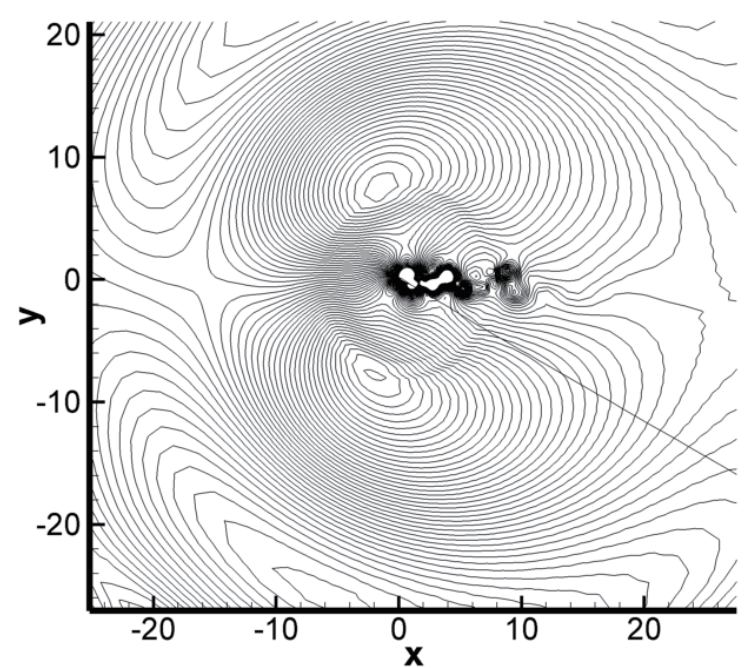

Fig. 5. Pressure distribution for the case of $f_{0}=0.15$ and $h_{0}=0.1$ and $\alpha=30^{\circ}$ 
as shown in entropy distribution. The last is pressure waves generated by Karman vortex shedding in the trailing edge.

Figs.7 shows an instantaneous distribution of the entropy for the cases of $f_{0}=0.15, h_{0}=0.1$ and several angles of attack in the wake flow. According to Young \& Lai [8], the entropy distribution simulates the vortex flow very well. The figure shows two or more mixed frequencies in the wake flow. The figures show that the distance between vortex cells decreases with the increase of angle of attack. From this fact, it can be predicted that the principal oscillation frequency decreases

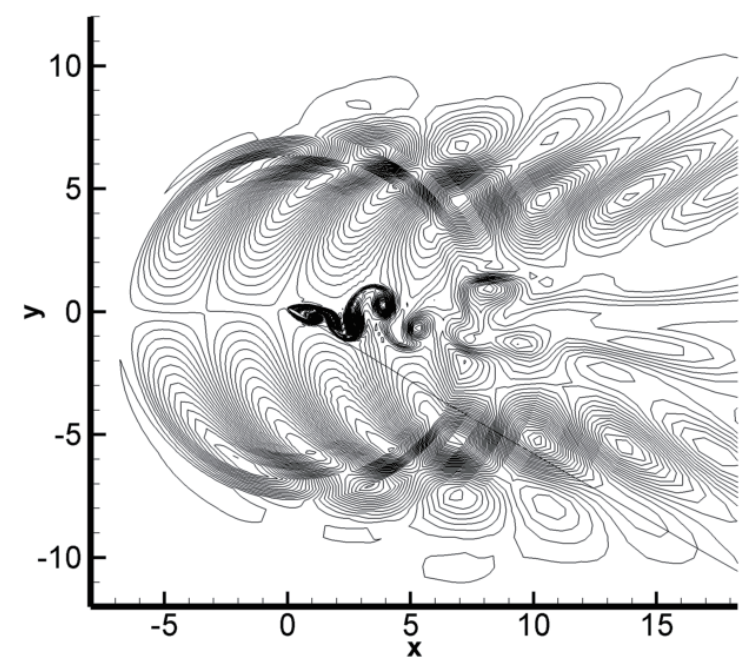

Fig. 6. Entropy distribution for the case of $f_{0}=0.15$ and $h_{0}=0.1$ and $\alpha=30^{\circ}$

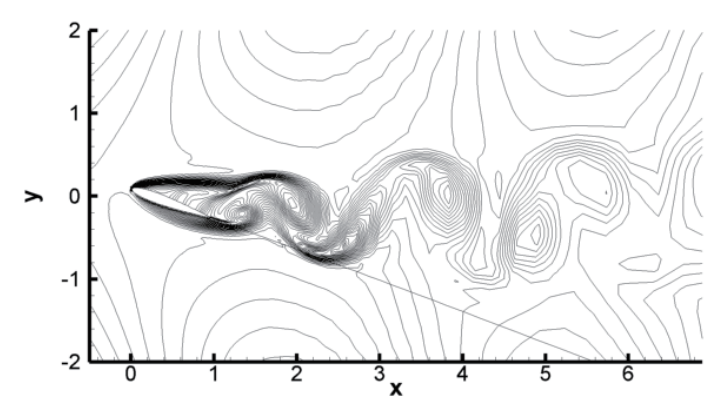

(a) $\alpha=20^{\circ}$

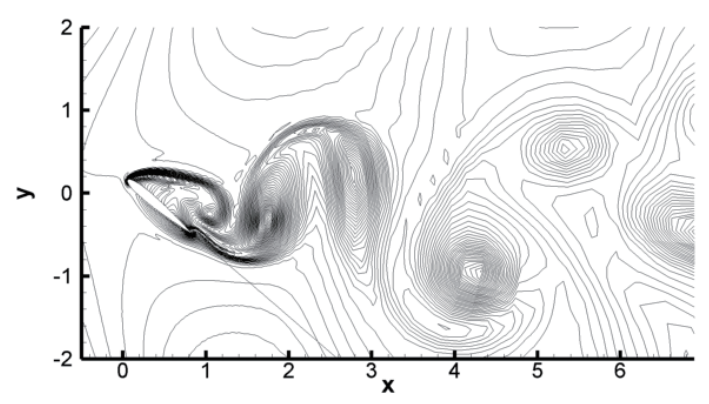

(c) $\alpha=40^{\circ}$

Fig. 7. Entropy distribution for the case of $f_{0}=0.15$ and $h_{0}=0.1$ with the increase of angle of attack. Figs. 8 and 9 show the entropy and the vorticity distributions during one cycle for the case of $f_{0}=0.1, h_{0}=0.2$ and $\alpha=50^{\circ}$. The figures show the separated flow generated at the upper surface of the airfoil as well as the vortex flow.

The frequency characteristics were analyzed for the lift coefficient. In Figs.10, the PSD distributions of lift coefficients are plotted for several cases of oscillation frequencies and amplitudes. According to the results shown in Fig.4, the principal oscillation frequency occurs due to the forced vibration and the angle of attack. The high frequency appears at low angle of attack while the low frequency appears at high angle of attack. The figures show the principal oscillation frequencies depending on the angle of attack, irrespective of the forced oscillation frequency and the amplitude. When the angle of attack is $30^{\circ}$, the principal oscillation frequencies appear with similar intensities at the frequencies of $f=0.35$ and 0.175 , which is shown as more complex change in the lift coefficients in Fig. 4 under dynamic stall. The principal oscillation frequency decreases with the increase of angle of attack. This shows that the plunging frequency does not affect the frequency of basic vortex shedding. Fig.11 shows the principal oscillation frequency according to the angle of attack, which is independent of the oscillation amplitude and the plunging frequency. Fig. 11 confirms that the principal oscillation frequency decreases with the

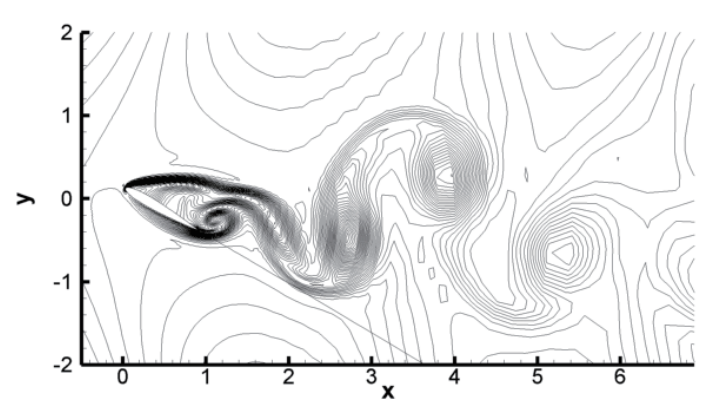

(b) $\alpha=30^{\circ}$

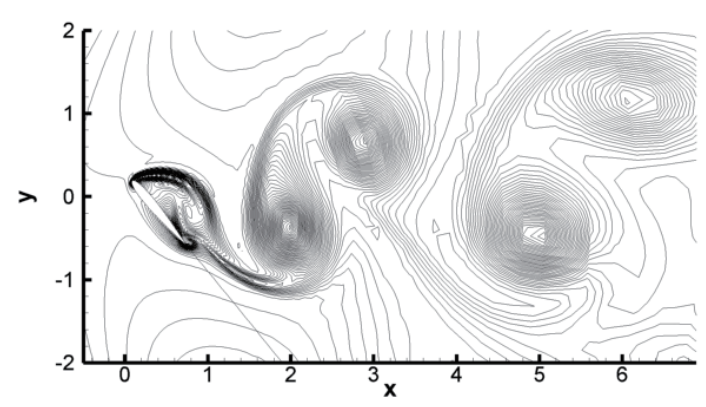

(d) $\alpha=50^{\circ}$ 
increase of angle of attack, as mentioned in Fig. 7. The flow conditions of Fig.7(d) and Fig.8(b) have same frequency, same amplitude and different angle of attack, the vortex

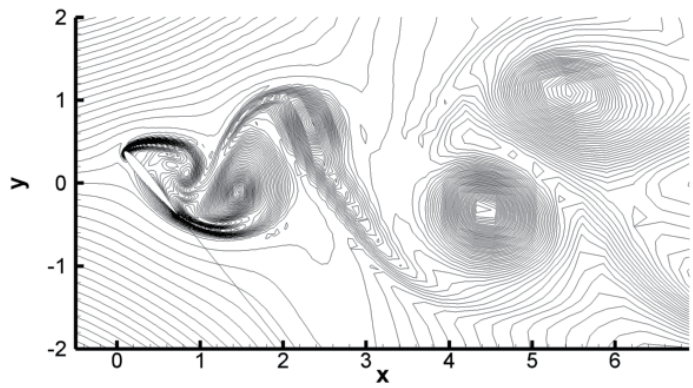

(a) $t=0$ cell flowed to a similar position. The distances between the vortex cells are similar to each other. This means that the principal oscillation frequency is similar to each other, as

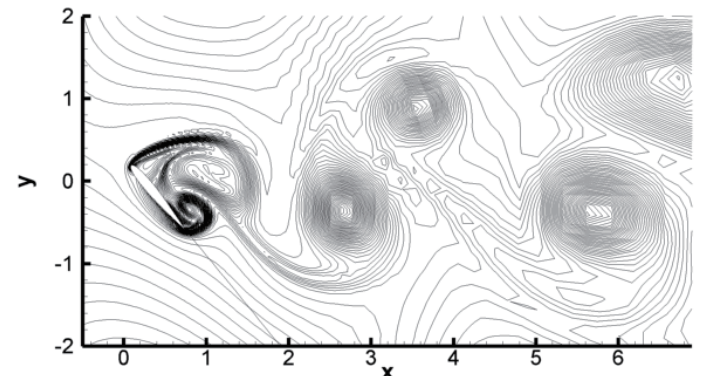

(b) $t=T p / 3$

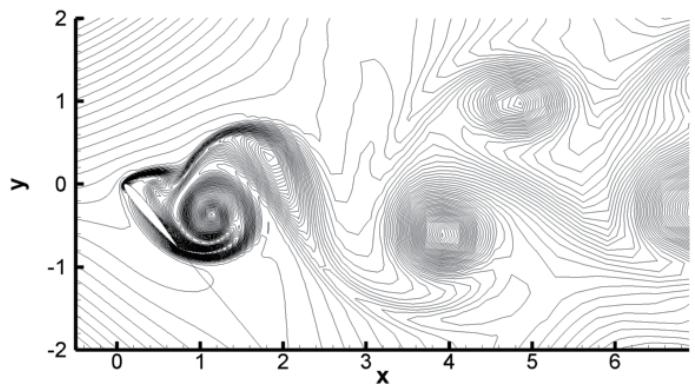

(c) $t=2 T p / 3$

Fig. 8. The vorticity distribution during one cycle for the case of $f_{0}=0.1$ and $h_{0}=0.2$ and $\alpha=50^{\circ}$

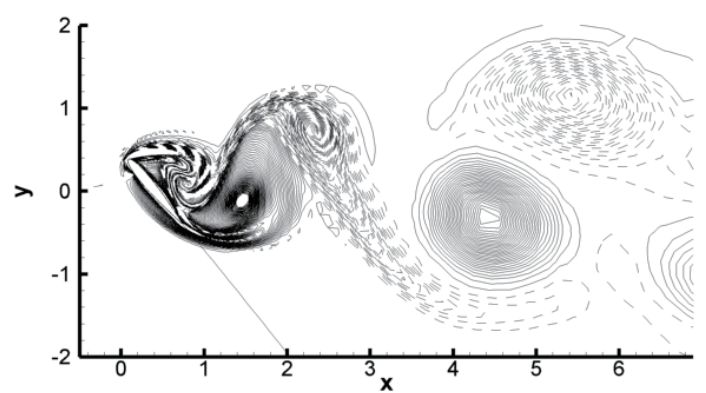

(a) $t=0$

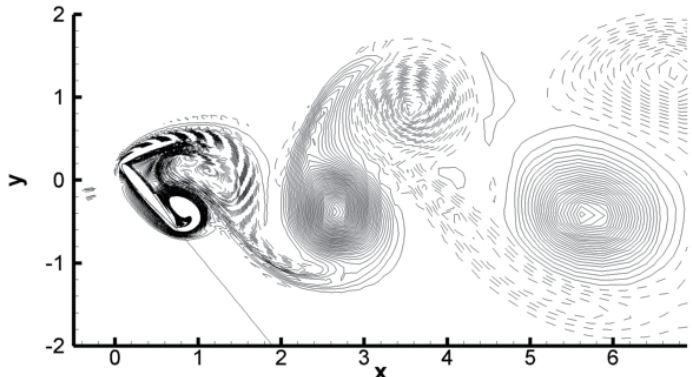

(b) $t=T p / 3$

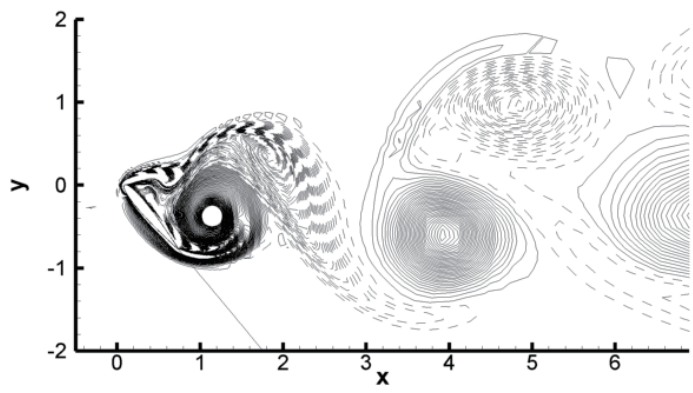

(c) $t=2 T p / 3$

Fig. 9. The vorticity distribution during one cycle for the case of $f_{0}=0.1$ and $h_{0}=0.2$ and $\alpha=50^{\circ}$ (solid line: counterclockwise, dashed line: clockwise) 


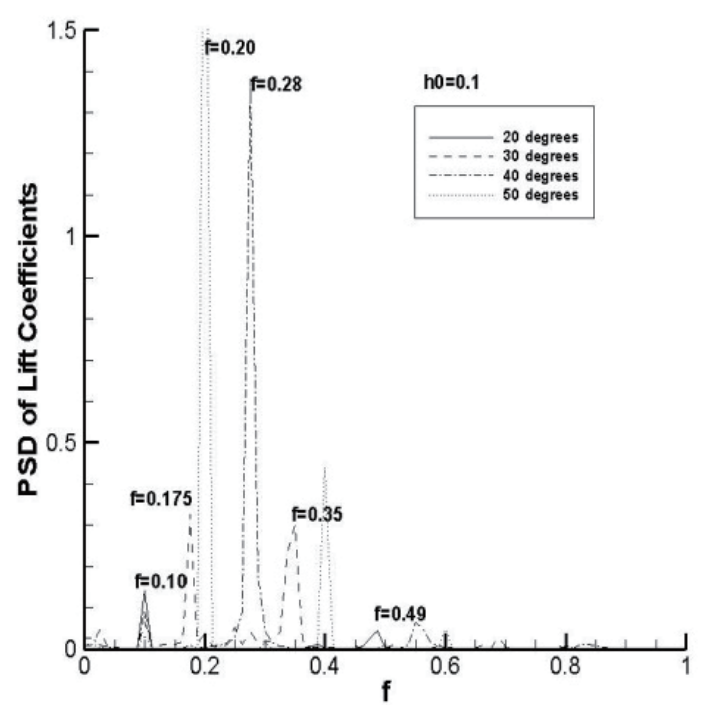

(a) for the case of $f_{0}=0.1$ and $h_{0}=0.1$

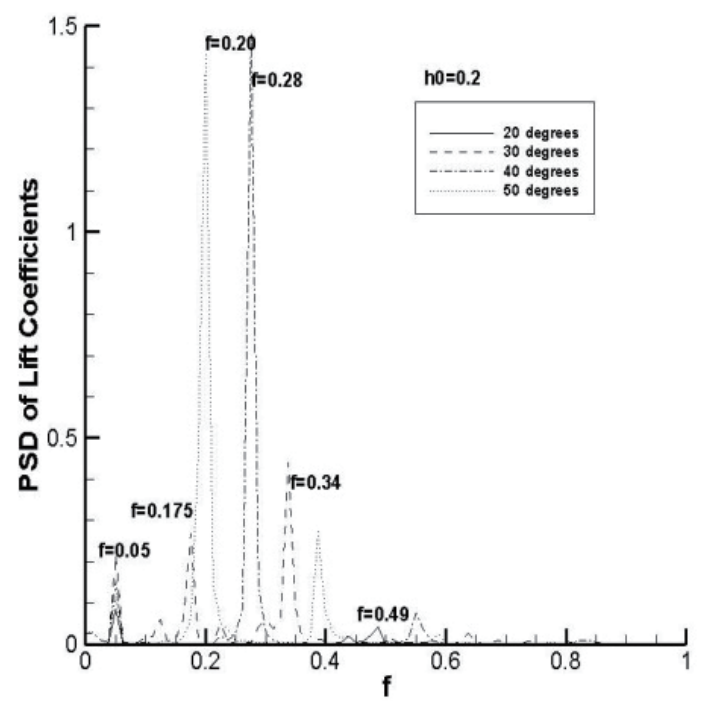

(c) for the case of $f_{0}=0.05$ and $h_{0}=0.2$

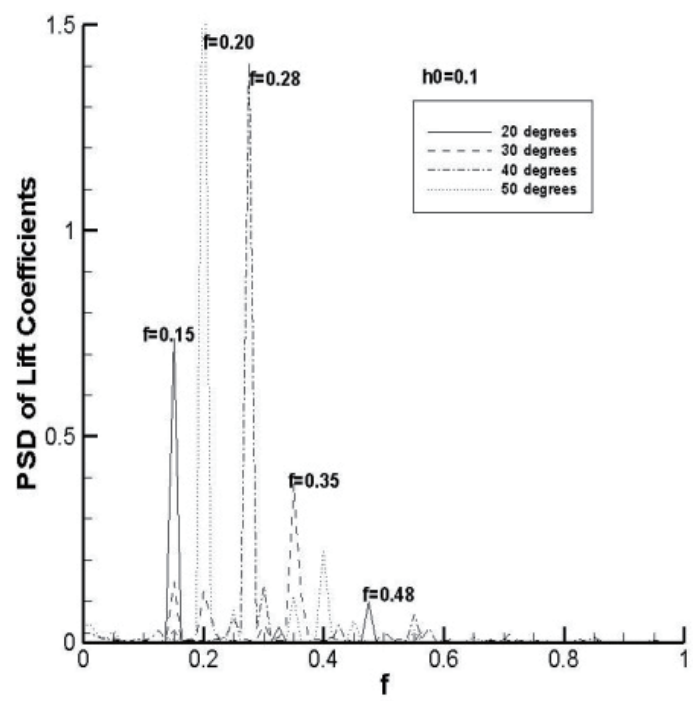

(b) for the case of $f_{0}=0.15$ and $h_{0}=0.1$

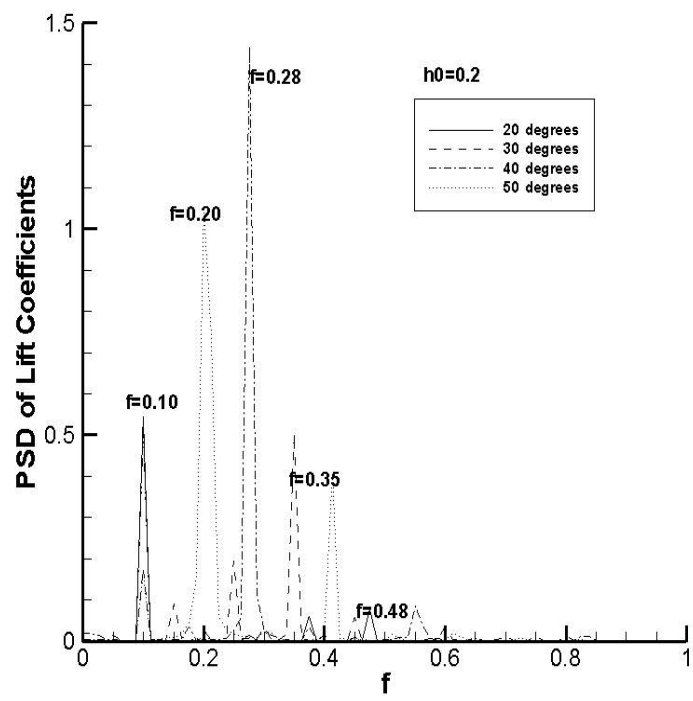

(d) for the case of $f_{0}=0.1$ and $h_{0}=0.2$

Fig. 10. PSD distribution of lift coefficients

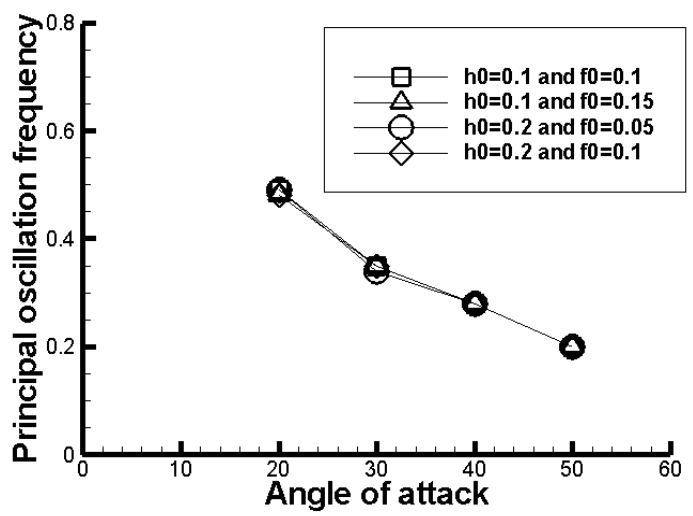

Fig. 11. Principal oscillation frequency according to the angle of attack 
Fig. 11 confirms.

\section{Conclusions}

Navier-Stokes equations with the LES turbulence model were calculated by the high-order high-resolution numerical scheme, termed the OHOC scheme, to analyze the plunging motion characteristics at a high angle of attack. The vortex shedding characteristics occurring in the wake were also analyzed, along with the characteristics of the wave propagating to the whole area, using a highresolution numerical scheme. The flow due to the plunging oscillation at high angle of attack has the characteristics of the waves generated by the plunging motion and vortex shedding. The periodic characteristics of the large separated flow on the airfoil surface are simple at the higher angle of attack but become complex at the lower angle of attack under dynamic stall. Using the frequency analysis of lift coefficients, the flow characteristics are determined by the principal oscillation frequencies due to the forced plunging oscillation and the separated flow. Irrespective of the forced oscillation frequency and the amplitude, the principal oscillation frequency according to the angle of attack is uniform.

\section{Acknowledgement}

This study was supported by research fund from Chosun University, 2013.

\section{References}

[1] Platzer, M.F., Jones, K.D., and Young J., "Flapping Wing Aerodynamics: Progress and Challemges", AIAA Journal, Vol. 46, No. 9, 2008, pp. 2136-2149.

[2] Isogai, K., Shinmoto, Y., and Watanabe Y., "Effects of Dynamic Stall on Propulsive Efficiency and Thrust of Flapping Airfoil", AIAA Journal, Vol. 37, No. 10, 1999, pp. 1145-1151.

[3] Birnbaum, W., "Das ebene Problem des Schlagenden Fluegels", Zeitschrift fuer Angewandte Mathematik und Mechanical, Vol. 4, No. 4, 1924, pp. 277-292.

[4] Garrick, I.E., "Propulsion of a Flapping and Oscillating Airfoils”, NACA Rept. 567, 1936.

[5] Jones, K.D., Dohring, C.M., and Platzer, M.F., "Experimental and Computational Investigation of the Knoller-Benz Effect”, AIAA Journal, Vol. 36, No. 7, 1998, pp.
1240-1246.

[6] Lai, J.C.S., and Platzer, M.F., "Jet Characteristics of a Plunging Airfoil”, AIAA Journal, Vol. 37, No. 12, 1999, pp. 1529-1537.

[7] Taylor, G.K., Nudds, R.L., and Thomas, A.L.R., "Flying and Swimming Animals Cruise at a Strouhal Number Tuned for High Power Efficiency", Nature (London), Vol. 425, Oct., 2003, pp. 707-711.

[8] Young, J., and Lai, J.C.S., "Oscillation Frequency and Amplitude Effects on the Wake of a Plunging Airfoil", AIAA Journal, Vol.42, No.10, 2004, pp. 2042-2052.

[9] Rival, D., and Tropea, C., "Characteristics of Pitching and Plunging Airfoils under Dynamic-Stall Conditions", Journal of Aircraft, Vol. 47, No. 1, 2010, pp. 80-86.

[10] Moryossef, Y., and Levy, Y., "Effect of Oscillations on Airfoils in Close Proximity to the Ground", AIAA Journal, Vol. 42, No. 9, 2004, pp. 1755-1764.

[11] Wagner, C., Huttl, T., and Sagaut, P., Large-Eddy Simulation for Acoustics, CAMBRIDGE UNIVERSITY PRESS, 2007.

[12] Wilcox, D.C., Turbulence Modeling for CFD, 2nd., DCW Industries Inc., California, 2000.

[13] Kim, J.W., and Lee, D.J., "Optimizes Compact Finite Difference Schemes with maximum Resolution”, AIAA Journal, Vol. 34, No. 5, 1986, pp. 887-893.

[14] Sandberg, R.D., Jones, L.E., and Sandham, N.D., "A Zonal Characteristic Boundary Condition for Numerical Simulations of Aerodynamic Sound", ECCOMA CFD, 2006.

[15] Kim, T.S., and Kim, J.S., "Numerical Analysis of the Three Dimensional Wake Flow and Acoustic Field around a Circular Cylinder", Int. J. of Aeronautical and Space Sciences, Vol. 11, No. 4, 2010, pp. 319 235.

[16] Blake, W.K., "Dipole Sound from Cylinders", Mechanics of Flow induced Sound and Vibration, Vol. 1, Academic Press, New York, 1986, pp. 219-287.

[17] Williamson, C.H.K., "Three Dimensional Wake Transition", Journal of Fluid Mechanics, Vol. 328, 1996, pp. 345-407.

[18] Yoo, J.K., and Kim, J.S., "Analysis of Unsteady Oscillating Flow around Two Dimensional Airfoil at High Angle of Attack", J. Comput. Fluids Eng., Vol. 18, No. 1, 2013, pp. 1 6.

[19] Liu, Y., Li, K., Zhang, J., Wang, H., and Liu, L., "Numerical Bifurcation Analysis of Static Stall of Airfoil and Dynamic Stall under Unsteady Perturbation", Commun Nonlinear Sci Numer Simulat, Vol. 17, No. 8, 2012, pp. 34273434.

[20] Critzos, C.C., Heyson, H.H., and Boswinkle, R.W., Aerodynamic Characteristics of NACA0012 Airfoil Section at the Angle of Attack from $0^{\circ}$ to 180", NACA TN 3361, 1955. 
[21] Craig, A.P., and Hansman, R.J., "An Experimental Low Reynolds Number Comparison of a Wortmann FX67-K170 Airfoil, a NACA0012 Airfoil, and a NACA 64-210 Airfoil in
Simulated Heavy Rain", NACA CR 181119, 1987.

[22] Hoerner, S.F., and Borst, H.V., Fluid Dynamic Lift, Horerner Fluid Dynamics, U.S.A., 1985, pp. 2-17 4-26. 